\title{
LA COMPRENSIÓN SINTÁCTICA EN LA AFASIA. UNA HERRAMIENTA DE EVALUACIÓN CON CLÁUSULAS RELATIVAS
}

\author{
SYNTACTIC COMPREHENSION IN APHASIA. \\ AN EVALUATION TEST WITH RELATIVE CLAUSES
María Elina Sánchez ${ }^{1}$, Martín Fuchs ${ }^{1,2}$, Analí Taboh ${ }^{1}$, Juan Pablo Barreyro ${ }^{3,4}$ Y Virginia Jaichenco ${ }^{1}$ ${ }^{1}$ Instituto de Lingüística, Facultad de Filosofía y Letras, Universidad de Buenos Aires (FFyL, UBA). Argentina ${ }^{2}$ Department of Linguistics. Yale University. USA \\ ${ }^{3}$ Facultad de Psicología, Universidad de Buenos Aires (FPsi, UBA). Argentina \\ ${ }^{4}$ Consejo Nacional de Investigaciones Científicas y Técnicas (CONICET). Argentina. \\ mesanchez@filo.uba.ar
}

Enviado: $16 / 08 / 2017$

Aceptado: 21/01/2018

\begin{abstract}
Resumen
Este trabajo tuvo con objetivo probar un instrumento de evaluación de los déficits en la comprensión de oraciones de sujetos con afasia (SCA) en español e investigar si permite distinguir perfiles entre pacientes. Se diseñó una tarea de emparejamiento oración-dibujo y se manipuló el tipo de estructura sintáctica: oraciones con cláusulas relativas de sujeto (RS- El gato que muerde al conejo es rosa) y de objeto (RO- El gato al que muerde el conejo es rosa). La prueba se administró a 151 hablantes nativos de español, divididos en 3 grupos de edad y en 3 niveles de escolaridad como grupo control y a 5 SCA. Los resultados mostraron que en el grupo control existe una
\end{abstract}

\begin{abstract}
This work aimed to test an instrument for the assessment of deficits in sentence comprehension in Spanish and to investigate if it allows distinguishing profiles of individual with aphasia (IWA). A binary sentence-picture matching task was designed and the type of syntactic structure was manipulated: sentences with subject relative clauses (RS- El gato que muerde al conejo es rosa [The cat that bites the rabbit is pink]) and with object relative clauses (RO- El gato al que muerde el conejo es rosa [The cat that the rabbit bites is pink]). The study was administered to 151 native Spanish speakers, divided into 3 age groups and 3 levels of schooling (control
\end{abstract}

Para citar este artículo / To cite this article: Sánchez, María Elina; Fuchs, Martín; Taboh, Analí; Barreyro, Juan Pablo y Virginia Jaichenco (2018). La comprensión sintáctica en la afasia. Una herramienta de evaluación con cláusulas relativas. ELUA, 32: 317-329. doi: 10.14198/ELUA2018.32.14

Enlace / Link: http://dx.doi.org/10.14198/ELUA2018.32.14 
fuerte interacción entre el tipo de oración y el nivel de escolaridad, con más errores en las oraciones RO a medida que disminuye el nivel de escolaridad. Tres SCA (AG, RD y $\mathrm{RR})$ se diferenciaron de su grupo de control sólo en las RO, un SCA (OV) en ambas estructuras y el sujeto RC rindió igual que su grupo control en los dos tipos de oraciones. Los datos nos permiten discutir la sensibilidad de la prueba para detectar alteraciones específicas de la comprensión sintáctica de personas con afasia hablantes del español y discriminar pacientes con diferentes perfiles de afasia.

PALABRAS CLAVE: Afasia; agramatismo; comprensión sintáctica; oraciones relativas; Hipótesis de la supresión de la huella; evaluación. group), and 5 IWA. The results showed that in the control group there is an interaction between the type of structure and the level of schooling, with more errors in RO as schooling decreases. Three IWA (AG, RD and RR) differed their corresponding controls only in the ORs, one IWA (OV) differed from the corresponding controls in both structures, and there is no significant difference in performance between subject RC and the corresponding controls for both types of sentences. Data allow us to discuss the sensitivity of the test to detect specific alterations of syntactic comprehension in individuals with aphasia speakers of Spanish and discriminate patients with different types of aphasia.

KEYWORDS: Aphasia; agrammatism; sentence comprehension; relative clauses; Trace Deletion Hypothesis; evaluation.

\section{INTRODUCCIÓN}

La afasia adquirida es la alteración de algún aspecto del procesamiento del lenguaje como consecuencia de una lesión cerebral. Dada la complejidad del lenguaje, se puede observar un vasto número de tipos de afasia, cada uno de ellos como resultado de una forma particular de daño que compromete a un componente o a una combinación de componentes del sistema que subyace al procesamiento (Caramazza, 1984). Entre las diversas alteraciones del lenguaje producidas por una lesión cerebral, aquellas que se relacionan con un déficit de las habilidades sintácticas se conocen como agramatismo. Si bien inicialmente el agramatismo se relacionó solamente con dificultades en la producción, se ha establecido a partir del trabajo pionero de Zurif y Caramazza (1976) que se manifiesta también con dificultades en la comprensión de oraciones.

Se han propuesto distintas explicaciones acerca de cuál es la causa de los problemas de comprensión en el agramatismo, pero la mayoría de las hipótesis presenta consenso respecto de que la alteración se hace visible frente a las oraciones con orden no canónico. El orden de los elementos en la oración codifica información sintáctica: constituye uno de los aspectos que indican qué hizo qué a quién en el evento descrito por una frase. Cada lengua se caracteriza por tener un orden canónico, esto es, una forma básica, no marcada, de ordenar los constituyentes en la oración; por ejemplo, en español el orden canónico es Sujeto-Verbo-Objeto (SVO). Este orden se corresponde con una asignación de roles temáticos. El verbo codifica información temática (semántica) y le asigna a cada elemento que lo acompaña un rol o papel. Por ejemplo, un verbo transitivo como "golpear" necesita dos argumentos: un AGENTE o quién realiza la acción y un PACIENTE que es quien recibe la acción y sufre modificaciones. Hay verbos que requieren 1 argumento (intransitivos) o 3 argumentos (ditransitivos).

Volviendo al orden estructural, cuando una oración sigue el orden canónico, la asignación de los roles temáticos (y la consecuente comprensión del evento) puede hacerse 
en forma lineal (AGENTE-ACCIÓN-TEMA/ PACIENTE para SVO). Cuando se aparta de dicho orden, en algunos casos es aún posible hacer una correcta asignación de roles temáticos si los participantes tienen rasgos semánticos distintos que solo habilitan una interpretación (por ejemplo, en la oración La comida es devorada por el perro, es posible que el perro devore la comida y no al revés). Ahora bien, cuando una oración se aparta del orden canónico y es semánticamente reversible (cualquiera de los participantes puede realizar o recibir la acción, por ejemplo, El niño patea al señor), entonces es necesario recurrir a otro tipo de información sintáctica.

En el caso de los sujetos con afasia (SCA) agramática, la comprensión suele estar caracterizada por un efecto de canonicidad, esto es, presentan más dificultades para aquellos tipos de oraciones semánticamente reversibles cuyo orden de constituyentes se aparta del orden canónico (Penke, 2011). Los SCA agramática suelen asignar el papel temático AGENTE al primer sintagma nominal que aparece en la oración según una estrategia lineal, lo que produce una comprensión correcta en ciertos tipos de estructuras, pero no en otras (Caplan 1983; Grodzinsky 1986; 1990). Así, estos sujetos presentan generalmente un buen rendimiento en las oraciones activas (La abuela besa a la niña), en las oraciones con cláusulas relativas de sujeto (La abuela que besa a la niña es cariñosa) y en las oraciones dislocadas de sujeto (Es la abuela la que besa a la niña), pero presentan un rendimiento inadecuado en las oraciones pasivas (La niña es besada por la abuela), en las oraciones con cláusulas relativas de objeto (La niña a la que besa la abuela es cariñosa) y en las oraciones dislocadas de objeto (A la niña la besa la abuela), siempre que sean semánticamente reversibles.

\section{1. ¿Cómo se explica el perfil agramático en la comprensión de los sujetos con afasia?}

Diversas propuestas han intentado dar cuenta de los mecanismos alterados subyacentes en la comprensión de los SCA. En términos generales se las puede agrupar en dos líneas. Por un lado, aquellas que explican los déficits de los SCA como resultado exclusivamente de una limitación de la capacidad de procesamiento, relacionándolos con factores como la memoria y la atención (Just \& Carpenter, 1992; Miyake et al, 1994). Por otro lado, aquellas que relacionan los déficits con factores específicamente lingüísticos y los atribuyen a la alteración de algún componente representacional del procesamiento sintáctico (Caramazza \& Zurif, 1976; Caplan \& Futre, 1986; Grodzinsky, 1986; Linebarger, Schwartz \& Saffran 1983, entre otros). También se han intentado propuestas que conectan ambos tipos de explicaciones (Garraffa \& Grillo, 2008).

Entre aquellas que se concentran en factores específicamente lingüísticos, una hipótesis que ha sido extensamente discutida (Berreta et al. 2001; Caramazza et al. 2005; Gavarró \& Dotti 2014; Hanne et al. 2011; Miera \& Cuetos 1998; Piñango \& Burkhardt 2001, entre otros) y que permite explicar el rendimiento diferencial en distintos tipos de oraciones, es la llamada Hipótesis de Supresión de la Huella de Grodzinsky (1986, 2000). Esta hipótesis parte de presupuestos de la lingüística formal, específicamente del marco de la teoría de Rección y Ligamiento de Chomsky (1981) y también presupone la modularidad cognitiva, en especial en el procesamiento del lenguaje (Fodor 1983). Según esta teoría, las oraciones se generan en una estructura profunda y luego se materializan en una estructura superficial. Algunas oraciones sufren movimientos de elementos al pasar de una estructura a otra. 
Cuando se producen un desplazamiento o movimiento, por ejemplo, como sucede en las oraciones pasivas, todo elemento desplazado deja en su posición de origen otro elemento representacional, esto es, una huella del movimiento, coindizada con él. La coindización (ver en la Figura 1 como $h_{\mathrm{i}}$ ) permite luego la asignación de papeles temáticos en la estructura de superficie. La novedad introducida por Grodzinsky fue proponer que el déficit de la comprensión agramática se debe a la supresión de las huellas de la representación de la estructura superficial, lo que impide la asignación de papeles temáticos a los constituyentes desplazados. Por ejemplo, en una oración pasiva como El niño fue empujado por el bombero, el sintagma nominal o frase nominal ( $\mathrm{SN}$, constituyentes en los que el núcleo es un sustantivo) "el niño" es un argumento del verbo y se genera en la estructura profunda como su complemento. Luego, se mueve a la posición preverbal para ocupar la posición de sujeto oracional en la estructura de superficie y deja, en su posición original, una huella coindizada con él. En este contexto, una asignación correcta de los roles implica que la huella que recibe en la posición de complemento el papel temático de TEMA o PACIENTE, lo transmite al SN desplazado (sujeto oracional) a través de la cadena de coindización. Pero, si la huella de este movimiento está borrada, no hay elemento que reciba el papel temático de TEMA o PACIENTE y su asignación a "el niño" falla, dando lugar a una comprensión alterada.

Grodzinsky (2000) notó que este rendimiento defectuoso de los SCA agramática en las oraciones con movimiento sintáctico se produce a nivel de azar. Propuso entonces que, al encontrarse un SN sin papel temático, se aplica una estrategia (por defecto) no lingüística consistente en asignar los papeles temáticos linealmente, empezando por el de AGENTE. En las oraciones pasivas, por ejemplo, el objeto se ha desplazado a la posición preverbal, por lo que recibe el papel temático de AGENTE por la estrategia lineal. Pero el verdadero constituyente que es AGENTE de la oración también recibe este papel directamente de la preposición "por" en la estructura profunda, ya que las preposiciones también asignan roles temáticos. Frente a dos SN con papel AGENTE, el SCA agramática se ve forzado a elegir, e interpreta como AGENTE al primer SN algunas veces y al segundo SN, otras (ver Figura 1).

(a) Comprensión normal

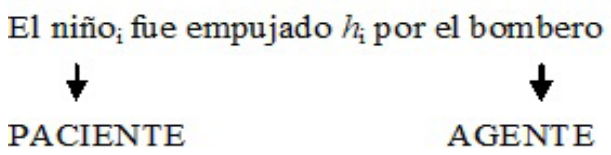

(b) Comprensión agramática

*El niño fue empujado (h) por el bombero

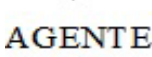
AGENTE $\longrightarrow$ NIVEL DE AZAR

Figura 1. Comprensión de oraciones pasivas: normales (a) vs. SCA agramática (b). 
En español, es usual encontrar que las pruebas que evalúan el procesamiento de oraciones utilizan distintos tipos de oraciones simples y complejas para conocer el desempeño de los SCA en la comprensión sintáctica (Goodglass, Kaplan \& Barbesi, 2001; López-Higes et al. 2004; Mendoza et al. 2005; Valle Arroyo \& Cuetos Vega, 1995, entre otras). Sin embargo, a menudo el uso de una diversidad de estructuras sintácticas no permite identificar puntualmente el problema sintáctico del paciente.

\subsection{Objetivos}

El objetivo de este trabajo fue poner a prueba la sensibilidad de una prueba que evalúa la comprensión sintáctica de oraciones con cláusulas relativas de sujeto y de objeto en un grupo de SCA e investigar si permite discriminar adecuadamente distintos perfiles de pacientes.

\section{MÉTODO}

\subsection{Participantes}

Cinco sujetos diagnosticados con afasia, 4 hombres y 1 mujer, participaron de este estudio (ver Tabla 1). El diagnóstico se realizó a través de una evaluación del lenguaje que incluía: el Test de Boston (Goodglass, Kaplan \& Barbesi, 2001) y el span de dígitos directo e inverso (Wechsler, 1987). Además se les administró una serie de pruebas de evaluación de la comprensión sintáctica diseñadas ad hoc que permitían evaluar la comprensión oral de oraciones con diferentes estructuras sintácticas (activas y pasivas) a partir de una tarea de señalamiento de imágenes (Jaichenco et al., 2012). En la Tabla 1 también se incluyen los datos de la localización cerebral de la lesión de cada uno de los sujetos. Todos los participantes del estudio, tanto los SCA como los que participaron como grupo control, firmaron un consentimiento previo por escrito.

\begin{tabular}{cccccccc}
\hline \multicolumn{6}{l}{ Sujeto Edad Género Dominancia manual Año de ACV Escolaridad } & Tipo de afasia & Lesión cerebral \\
\hline OV & 63 & M & D & 2003 & 17 años & Transcortical mixta & FTPO izquierda \\
AG & 58 & F & D & 2005 & 17 años & Broca & FTP izquierda \\
RD & 59 & M & D & 2000 & 14 años & Broca & TP PO izquierda \\
RR & 71 & M & D & 1997 & 17 años & Broca & FTP izquierda \\
RC & 59 & M & D & 1997 & 12 años & Conducción & FTP insular izquierda \\
\hline
\end{tabular}

Tabla 1. Datos demográficos, tipo de afasia y localización de la lesión cerebral de cada paciente.

\subsection{Materiales y procedimiento}

Se diseñó una tarea de emparejamiento oración-dibujo formada por 40 oraciones complejas que incluían una cláusula relativa, basada en Friedmann (2008). Del total, 20 contenían una cláusula relativa de sujeto (RS. Ej.: El gato que muerde al conejo es rosa) 
y 20 una relativa de objeto (RO. Ej.: El gato al que muerde el conejo es rosa). Todas las cláusulas contenían un verbo transitivo y los dos participantes que se vinculaban por esa acción tenían el mismo género (masculino o femenino) y mismo número (singular). Los estímulos se distribuyeron en dos sesiones. El examinador le leía al participante una oración y al mismo tiempo mostraba dos dibujos en la pantalla de una computadora. Un dibujo representaba la oración y el otro tenía invertidos los roles temáticos de los participantes de la acción. Esto es: si la oración blanco era: El gato que muerde al conejo es rosa, se mostraba un dibujo en el que un gato muerde a un conejo y otro en el que un conejo muerde a un gato. Ambos de color rosa. El participante debía escuchar la oración y señalar el dibujo correspondiente. La prueba contaba con cuatro ítems de práctica. Las respuestas de los sujetos fueron consignadas por el examinador y luego clasificadas como acierto o error.

Con esta prueba se evaluó también a un grupo control de 151 sujetos adultos sin alteraciones neurológicas, divididos en 3 grupos de edad y 3 niveles de escolaridad. El objetivo en este caso, dado que no era una prueba estandarizada, fue que cada paciente pudiera ser comparado con la media de su grupo control, en edad y nivel de escolaridad.

\subsection{Análisis de datos}

Se llevaron a cabo cinco análisis de varianza según el modelo ANOVA de dos factores 2 x 3s, tomando como variables independientes los tipos de oraciones (RO vs. RS) y el grupo (Grupo Control con Nivel de Escolaridad Media-GC NEM, Grupo Control con Nivel de Escolaridad Alta-GC NEA y los valores de cada uno de los pacientes).

Para cada SCA se realizó un análisis comparativo con su grupo control emparejado por nivel de escolaridad. La edad no fue un factor a tener en cuenta, ya que en estudios anteriores se demostró que esta variable no tiene influencia en esta prueba (Sánchez et. al 2017). Se utilizó como herramienta analítica el programa SPSS en su versión 17 (SPSS 2008).

\section{RESULTADOS}

\subsection{Perfil de la comprensión del lenguaje de los pacientes afásicos}

En este apartado se reportarán los datos de las diferentes pruebas con las que se evaluó la comprensión del lenguaje, tanto de palabra aislada como del nivel oracional.

En la Tabla 2 se pueden observar los resultados del span de dígitos directo y de las pruebas de comprensión auditiva del Test de Boston. La Tabla 3 muestra los porcentajes de aciertos de cada uno de los SCA en oraciones activas ("El tigre patea al elefante") y pasivas ("El elefante es pateado por el tigre").

Los sujetos OV, AG, RD y RR presentan un perfil similar: un rendimiento relativamente adecuado en la comprensión de oraciones activas con dificultades en la mayoría de las pruebas de comprensión y de memoria, medida a través del span de dígitos directo. $\mathrm{OV}$ muestra adicionalmente problemas más severos en la comprensión de palabras aisladas y un span muy descendido. El sujeto RC no presenta dificultades severas de la comprensión del lenguaje. Muestra resultados adecuados en las tareas de comprensión sintáctica, una 
comprensión de palabras intacta y un span de dígitos alto. En su conjunto, los datos obtenidos en estas pruebas nos permitieron establecer el perfil lingüístico de cada uno de los sujetos y compararlos a la luz de los resultados de la prueba de comprensión de oraciones con cláusulas relativas.

\begin{tabular}{ccccc}
\hline \multirow{2}{*}{ Paciente } & \multirow{2}{*}{ Span de dígitos } & \multicolumn{3}{c}{ Comprensión auditiva Test de Boston } \\
\cline { 3 - 5 } & & Ejecución de órdenes & Palabras & Oraciones \\
\hline OV & 2 & $12 / 15$ & $28.5 / 37$ & $3 / 10$ \\
AG & 3 & $12.5 / 15$ & $29 / 37$ & $8 / 10$ \\
RD & 4 & $14 / 15$ & $35 / 37$ & $8 / 10$ \\
RR & 3 & $13 / 15$ & $36 / 37$ & $6 / 10$ \\
RC & 4 & $14 / 15$ & $36 / 37$ & $10 / 10$ \\
\hline
\end{tabular}

Tabla 2. Resultados de los SCA del span de dígitos y de la comprensión del Test de Boston

\begin{tabular}{ccc}
\hline \multirow{2}{*}{ Paciente } & \multicolumn{2}{c}{ Comprensión de oraciones } \\
\cline { 2 - 3 } & Activa & Pasiva \\
\hline OV & 87.5 & 59.38 \\
AG & 92.19 & 46.88 \\
RD & 95.31 & 46.88 \\
RR & 98.44 & 43.75 \\
RC & 87.5 & 87.5 \\
\hline
\end{tabular}

Tabla 3. Porcentaje de acierto de los SCA en la prueba diseñada ad hoc con oraciones activas y pasivas

\subsection{Comprensión de oraciones con cláusulas relativas}

La Tabla 4 muestra la media de acierto y la desviación típica de los cinco SCA que participaron de este estudio. En la nota de la Tabla 4 se puede ver la media y el desvío estándar de cada grupo control con los que se comparó a los pacientes (Grupo Control de Escolaridad Media-GC EM y Grupo Control de Escolaridad Alta-GC EA) en las dos estructuras sintácticas. El análisis de varianza detecta diferencias significativas entre las dos estructuras sintácticas $F_{(1,38)}=69.01 ; p<.001$, las estructuras RS presentan mayor cantidad de aciertos que las RO. El análisis detecta diferencias significativas entre los grupos estudiados y los pacientes $F_{(6,228)}=12.52 ; p<.001$, y también muestra efectos de interacción entre los grupos y las estructuras de las oraciones $F_{(6,228)}=6.16 ; p<.001$. Tanto el grupo control con alto nivel de escolaridad como el de escolaridad media tienen un desempeño más adecuado que los SCA en los dos tipos de oraciones. Los análisis post-hoc, empleando las prueba de contraste de Bonferroni, muestran que mientras el grupo control 
con nivel de escolaridad alto (EA) no presenta diferencias entre las dos estructuras, el grupo con escolaridad media (EM) presenta diferencias significativas, con más errores en las oraciones RO $(p<.05)$. En este último grupo, la interacción entre el Tipo de oración y el Nivel de escolaridad es significativa.

\begin{tabular}{lcc}
\hline Sujetos & RO & RS \\
\hline OV & $.25(.44)^{*}$ & $.60(.5)^{*}$ \\
AG & $.25(.44)^{*}$ & $.80(.41)$ \\
RD & $.35(.48)^{*}$ & $.85(.36)$ \\
RR & $.05(.22)^{*}$ & $.95(.22)$ \\
RC & $.55(.51)$ & $.94(.22)$ \\
& & \\
\hline & & \\
Nota: & & \\
CGEM .66 (.08) RO & & \\
CGEM .89 (.04) RS & & \\
CGEA .85 (.05) RO & \\
CGEA .98 (.01) RS &
\end{tabular}

Tabla 4. Media y Desvío típico (entre paréntesis) de cada SCA en cada una de las estructuras: oraciones con cláusulas relativas de objeto (RO) y relativas de sujeto (RS).

Con un asterisco $\left(^{*}\right)$ se marca la significatividad de cada sujeto con su grupo control.

A continuación se detalla la comparación del desempeño de cada paciente con su grupo control emparejado por el nivel educativo. El sujeto OV rinde significativamente peor en las dos estructuras $(\mathrm{p}>.01)$ en comparación a su grupo control de escolaridad alta. Asimismo, la diferencia se incrementa aún más en las oraciones con RO; es decir, en las oraciones con RO presenta muchos más errores que los sujetos sin lesión cerebral (ver Figura 2).

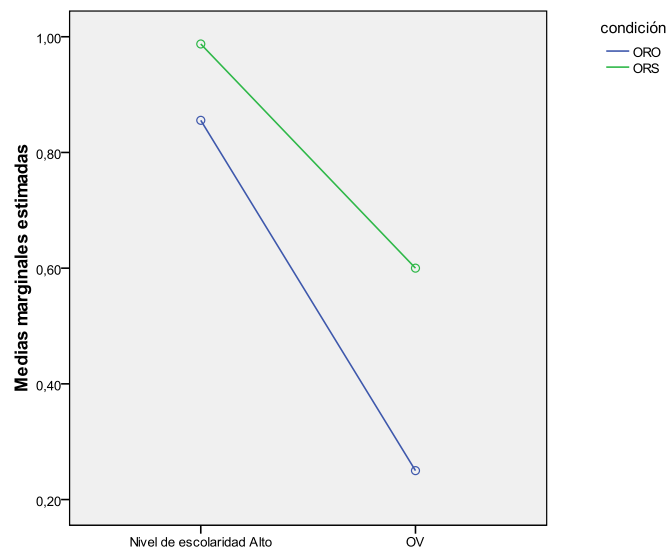

Figura 2. Comparación entre grupo control con escolaridad alta y paciente OV. En azul se ve el rendimiento en las oraciones con cláusula relativa de objeto (ORO) y en verde con cláusula relativa de sujeto (ORS). 
Los sujetos AG, RD y RR no se diferencian significativamente de su grupo control (escolaridad alta) en las oraciones con RS (ns.); sin embargo, el rendimiento en las oraciones con RO es significativamente más bajo $(p<.01)$ (ver Figura 3 (a), (b) y (c)).

(a) Paciente AG

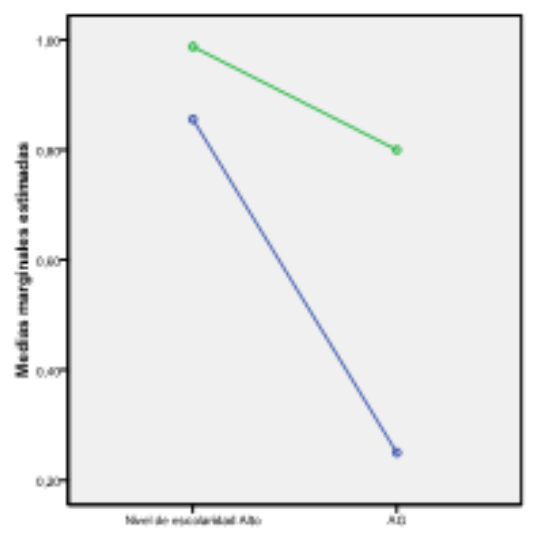

(b) Paciente RD
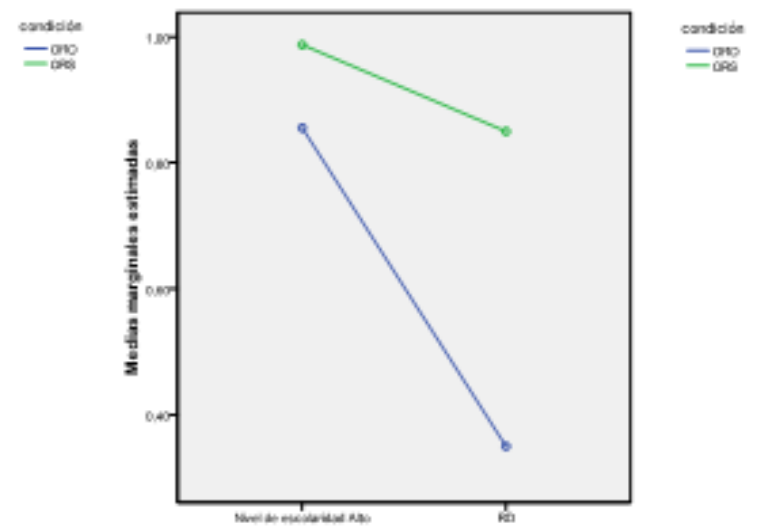

(c) Paciente RR

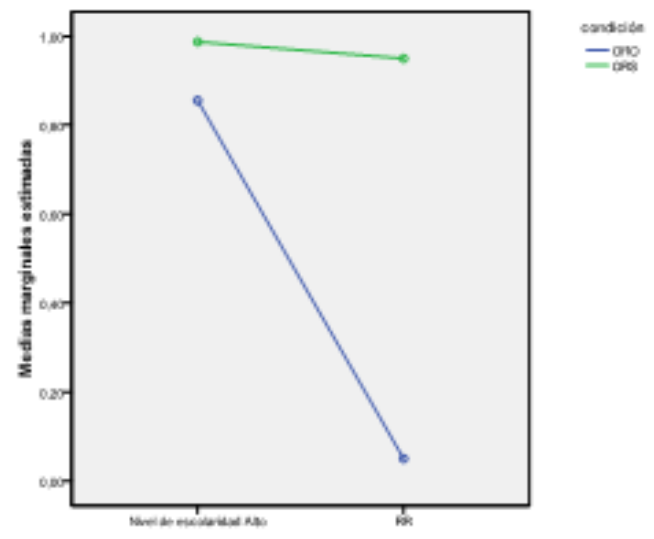

Figura 3. Comparación entre grupo control con escolaridad alta y sujetos AG, RD y RR. En azul se ve el rendimiento en las oraciones con cláusula relativa de objeto (ORO) y en verde con cláusula relativa de sujeto (ORS).

Por último, el sujeto RC no se diferencia significativamente de su grupo control con escolaridad media en ninguna de las dos estructuras (ns.) (ver Figura 4). 


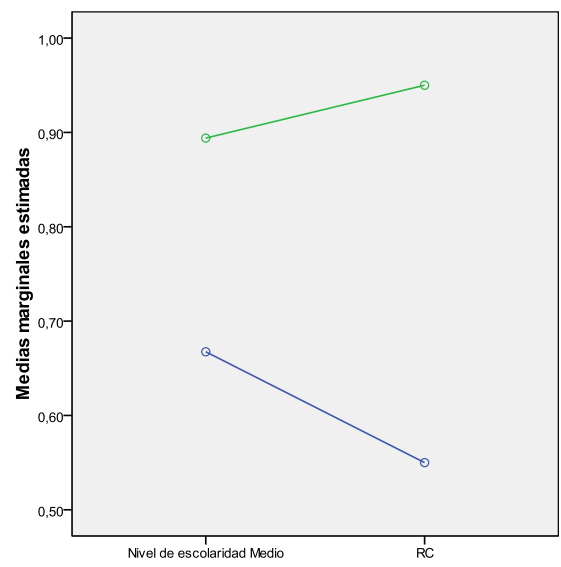

condición

二 ORO

Figura 4. Comparación entre grupo control con escolaridad media y paciente RC. En azul se ve el rendimiento en las oraciones con cláusula relativa de objeto (ORO) y en verde con cláusula relativa de sujeto (ORS).

\section{DISCUSIÓN}

El trabajo tuvo como objetivo principal poner a prueba la sensibilidad de una herramienta que evalúa la comprensión sintáctica de sujetos con afasia (SCA) utilizando dos tipos de oraciones diferentes: oraciones con cláusulas relativas de sujeto (El señor que lleva al niño es alto) y con relativas de objeto (El señor al que el niño lleva es alto). La prueba diseñada fue administrada a un grupo de cinco SCA y a un grupo control de sujetos sin lesiones cerebrales de diferentes niveles educativos y edades.

Los datos del grupo control han arrojado que la escolaridad es un factor que afecta directamente el rendimiento en este tipo de oraciones. A medida que el nivel de escolaridad desciende, el porcentaje de acierto de las estructuras también desciende, especialmente en las oraciones con cláusulas relativas de objeto. Para un análisis completo de la comparación entre el grupo control y un grupo de SCA ver Sánchez et al. (2017).

En el presente trabajo nos propusimos comparar el rendimiento en esta prueba de cada paciente con su grupo control, con el fin de poder establecer perfiles de SCA en base a los datos arrojados. Los resultados parecen mostrar, en primer lugar, que la prueba diseñada es un instrumento sensible, ya que permite distinguir entre SCA que presentan un perfil agramático y aquellos que no lo presentan. Los pacientes $\mathrm{OV}, \mathrm{AG}, \mathrm{RD}$ y RR (el primer paciente con afasia transcortical mixta y el resto con afasia de Broca) rinden muy por debajo en las oraciones con cláusulas relativas de objeto en comparación con su grupo control mostrando claramente el déficit en las habilidades sintácticas. En cambio, el sujeto $\mathrm{RC}$, con afasia de conducción, no presenta dificultades en el procesamiento sintáctico corroborando los datos arrojados en su evaluación del lenguaje. Cabe destacar que los resultados en la prueba con cláusulas relativas de este paciente analizados aisladamente podrían interpretarse como una alteración sintáctica; sin embargo, en comparación con su grupo control con escolaridad media, las diferencias no son significativas. Esto evidencia 
la necesidad de utilizar pruebas con datos de grupos control en los que el grado de escolaridad sea equivalente al de los pacientes, específicamente en los casos de estructuras sintácticas complejas. Asimismo, este sujeto tuvo un desempeño adecuado y parejo en la comprensión de oraciones tanto activas como pasivas, lo que confirma que no es un paciente con agramatismo.

La disociación entre oraciones relativas de objeto $(\mathrm{RO})$ y relativas de sujeto (RS) que presentan los sujetos AG, RD y RR, los tres diagnosticados con afasia de Broca, muestra claramente un efecto de canonicidad y un déficit de las alteraciones sintácticas. El rendimiento descendido de los sujetos agramáticos de este estudio en las oraciones relativas de objeto concuerda con la propuesta de la Hipótesis de la supresión de la huella (Grodzinsky 1995, 2000), en la que se establece que la alteración de los SCA se debe al borrado de las huellas, lo que impide la transmisión de los papeles temáticos desde las posiciones en las que se asignan a las posiciones desplazadas.

No debe perderse de vista la posibilidad de explicar estos resultados como lo propone un enfoque integral en el que se combine una explicación que provenga de la teoría lingüística, como la que acabamos de explicar, con una que atienda a aspectos más relacionados con el procesamiento, esto es, el hecho de que las estructuras que se apartan del orden canónico incrementan la carga en la memoria de trabajo, podría servir para interpretar los datos de los SCA de manera más completa (Garraffa \& Grillo 2008; Piñango \& Burkhardt 2001; Ruigendijk, Vasic \& Avrutin \& 2006). Los datos obtenidos en el presente trabajo parecen indicar que un enfoque que atienda al agramatismo como una alteración exclusivamente del procesamiento (Burkhardt, Piñango, \& Wong, 2003; Haarmann \& Kolk, 1991; para una revisión ver Dickey, Choy, \& Thompson, 2007), no explica acabadamente el déficit, ya que dos pacientes con el mismo span (4), RD y RC, muestran en las pruebas sintácticas un perfil completamente diferente. Es decir, estos datos permiten sumar evidencia a favor de las hipótesis representacionales que explican el rendimiento en la comprensión como una alteración lingüística.

En síntesis, la prueba presentada aquí resulta ser un instrumento de evaluación breve y de fácil administración. Este instrumento está pensado para evaluar específicamente la comprensión de oraciones y resultó ser sensible para establecer perfiles de sujetos con afasia, esto es, permite diferenciar a pacientes con alteraciones sintácticas de aquellos que no las presentan. El trabajo futuro se direccionará en seguir comparando los datos de SCA hablantes de español con los diferentes grupos controles. Además, sería interesante incluir este instrumento dentro de una batería más general de evaluación de la morfosintaxis en español.

\section{Referencias bibliográficas}

Beretta, A., Schmitt, C., Halliwell, J., Munn, A., Cuetos, F., \& Kim, S. (2001). The effects of Scrambling on Spanish and Korean agrammatic interpretation: Why linear models fail and structural models survive. Brain and Language, 79, 407-425.

Burkhardt, P., Piñango, M. M., \& Wong, K. (2003). The role of the anterior left hemisphere in realtime sentence comprehension: Evidence from split intransitivity. Brain and Language, 86, 9-22.

Caplan, D. \& Futre, C. (1986). Assignment of thematic roles by an agrammatic aphasic patient. Brain \& Language, 27, 117-135.

Caplan, D. (1983). A note on the "word order problem" in agrammatism. Brain and Language, 20, 155-165. 
Caramazza, A., Capasso, R., Capitani, E. and Miceli, G. (2005). Patterns of comprehension performance in agrammatic Broca's aphasia: A test of the Trace Deletion Hypothesis. Brain and Language, 94: 43-53

Caramazza, A. (1984). The logic of neuropsychological research and the problem of patient classification in aphasia. Brain and Language, 21, 9-20.

Chomsky, N. (1981). Lectures on Government and Binding. Dordrecht, Holanda: Foris.

Dickey, M. W., Choy, J. J., \& Thompson, C. K. (2007). Real-time comprehension of wh- movement in aphasia: Evidence from eyetracking while listening. Brain and Language, 100, 1-22

Ferreres, A.; Grus, J.; Jacubovich, S.; Jaichenco, V.; Kevorkian, A.; Piaggio, V.; Politis, D.; Recio, F. (1999). Batería para el Análisis de los Déficits Afásicos. Buenos Aires: JVE ediciones. Versión en español de la Battería per l'analisi dei deficit afasici de G. Miceli, A. Laudanna, C. Burani y C. Capasso. Berdata: Milan, 1991.

Friedmann, N. (2008). Traceless relatives: Agrammatic comprehension of relative clauses with resumptive pronouns. Journal of Neurolinguistics, 21(2), 138-149

Fodor, J. A. (1983). The modularity of mind. Cambridge, MA: MIT Press

Garraffa, M. \& Grillo, N. (2008). Canonicity effects as grammatical phenomena. Journal of Neurolinguistics, 21, 177-197.

Gavarró, A. \& H. Dotti. (2014). Long and short passives in Catalan and Spanish. Aphasiology 28(1): $82-98$

Goodglass, H., Kaplan, E. \& Barbesi, B. (2001) Evaluación de la Afasia y de Trastornos Relacionados (Test de Boston) $3^{\circ}$ Edición. Editorial Médica Panamericana, Madrid, 2005.

Grodzinsky, Y. (1986). Language deficits and the theory of syntax. Brain and Language, 27, 135-159.

Grodzinsky, Y. (1990). Theoretical perspectives on language deficits. Cambridge, MA: The MIT Press.

Grodzinsky, Y. (1995). Trace deletion, theta-roles, and cognitive strategies. Brain \& Language, 51, 467-497.

Grodzinsky, Y. (2000). The neurology of syntax: language use without Broca's area. Behavioral and Brain Sciences, 23(1), 1-71.

Haarmann, H. J., \& Kolk, H. H. (1991). Syntactic priming in Broca's aphasics: Evidence for slow activation. Aphasiology, 5, 247-263

Hanne, S.; Sekerina I.A.; Vasishth S.; Burchert, F.; \& De Bleser, R. (2011). Chance in agrammatic sentence comprehension: What does it really mean? Evidence from eye movements of German agrammatic aphasic patients. Aphasiology. 25:221-244.

Jaichenco, V.; Y. Sevilla, M.E. Sánchez, M. Maldonado \& Y. Grodzinsky (2012). Comprensión de clíticos en un caso de afasia agramática. Póster presentado en el X Congreso Argentino de Neuropsicología. Buenos Aires, Argentina.

Just, M. A., \& Carpenter, P. A. (1992). A capacity theory of comprehension: Individual differences on working memory capacity. Psychological Review, 99, 122-149.

Linebarger, M.C., Schwartz, M.F. \& Saffran, E.M. (1983). Sensivity to grammatical structure in socalled agrammatic aphasic. Cognition, 13, 361-392.

López-Higes, R.; Del Río, D.; Fernández Guinea, S., \& Martín, M.T. (2004). Exploración de la comprensión de oraciones a través de la batería ECCO XXIV Congreso Internacional de Logopedia, Audiología, Foniatría y Psicología del Lenguaje, pp. 673-684

Mendoza, E.; Carballo, G.; Muñoz, J.; \& Fresneda, M.D. (2005). CEG (Test de Comprensión de Estructuras Gramaticales). TEA.

Miera, G. \& Cuetos, F. 1998. Understanding disorders in agrammatic patients: capacity or structural deficits? Brain and Language, 64(3), 328-338

Miyake, A., Carpenter, P. y Just, M. A. (1994). A capacity approach to syntactic comprehension disorders: Making normal adults perform like aphasic patients. Cognitive Neuropsychology, 12(6), 651-679.

Penke, M. (2011). Syntax and language disorders. En Tibor Kiss \& Artemis Alexiadou (Eds.). Syntax: An international handbook. Berlín: Walter de Gruyter. 
Piñango M.M., \& Burkhardt, P. (2001). Pronominals in Broca's aphasia comprehension: The consequence of syntactic delay. Brain and Language. 79:167-168.

Ruigendijk, E., Vasic', N., \& Avrutin, S. (2006). Reference assignment: Using language breakdown to choose between theoretical approaches. Brain and Language, 96, 302-317.

Sánchez, M. E.; Taboh, A.; Fuchs, M.; Barreyro, J. P. \& Jaichenco, V. (2017). Comprensión de oraciones con cláusulas relativas. Un estudio comparativo entre sujetos con y sin alteraciones del lenguaje. Panamerican Journal of Neuropsychology-Cuadernos de Neuropsicología. Vol. 11. Número 3. Sep-Dic DOI: 10.7714/CNPS/11.3.211

Valle Arroyo, F. \& Cuetos Vega, F. (1995). EPLA. Evaluación del Procesamiento Lingüístico en la Afasia. Versión española de Psycholinguistics Assessments of Language Processing in Aphasia (PALPA) de Coltheart, M., Kay, J., Lesser, R.: Lawrence Erlbaum Associates, 1992

Wechsler, D. (1987). Wechsler Memory Scale-Revised. San Antonio, TX: The Psychological Corporation.

Zurif, E., \& Caramazza, A. (1976). Psycholinguistic structures in aphasia: Studies in syntax and semantics. In H. Whitaker, \& H. A. Whitaker (Eds.), Studies in neurolinguistics, Vol. I. New York: Academic Press. 
